\title{
Model Approach in Heterogeneous Catalysis: Kinetics and Thermodynamics of Surface Reactions
}

\author{
Published as part of the Accounts of Chemical Research special issue "Microscopic Insights into Surface Catalyzed \\ Chemical Reactions".
}

\author{
Swetlana Schauermann ${ }^{*} \dagger, \dagger$ and Hans-Joachim Freund ${ }^{\dagger}$ \\ ${ }^{\dagger}$ Fritz-Haber-Institut der Max-Planck-Gesellschaft, Faradayweg 4-6, 14195 Berlin, Germany \\ ${ }^{\ddagger}$ Institut für Physikalische Chemie, Christian-Albrechts-Universität zu Kiel, Max-Eyth-Strasse 1, 24118 Kiel, Germany
}

\section{Supporting Information}

CONSPECTUS: Heterogeneous catalysts are widely employed in technological applications, such as chemical manufacturing, energy harvesting, conversion and storage, and environmental technology. Often they consist of disperse metal nanoparticles anchored onto a morphologically complex oxide support. The compositional and structural complexity of such nanosized systems offers many degrees of freedom for tuning their catalytic performance. However, a rational design of heterogeneous catalysts based on an atomistic-level understanding of underlying surface processes has not been fully achieved so far and remains one of the primary goals for catalysis research. In our group, we developed concepts for replacing highly complex real supported catalysts by simplified model systems, which complexity can be gradually increased in order to mimic certain structural aspects of practically relevant catalysts in a controlled way. Well-defined model systems consisting of metalnanoparticle ensembles supported on planar oxide substrates have proven to provide a successful approach to achieve fundamental insights into heterogeneous catalysis.

In this Account, two mechanistic case studies focusing on an atomistic-level understanding of surface chemistry are presented in which we investigate how the nanoscopic nature of metal clusters affects their interaction with the adsorbates and the reactive processes. Particularly, we investigate the effects of the particle size and the flexibility of the atoms constituting metal clusters on the binding energy of gas-phase adsorbates, such as $\mathrm{CO}$ and oxygen. We identified two major structural factors determining the binding energy of gas phase adsorbates on metal nanoparticles: the local configuration of the adsorption site and the particle size. While the effect of the local configuration of the adsorption site was found to be adsorbate specific, the reduction of the cluster size results in a pronounced decrease of binding energy for both adsorbates and appears to be a general trend.

In the second case study, we address the role of the surface modifiers, such as carbon, on the process of hydrogen diffusion into volume of Pd nanoparticles that was previously identified is an important step in hydrogenation chemistry. We provide for the first time direct experimental evidence that, inline with the recent theoretical predictions, the atomically flexible low-coordinated surface sites on Pd particles play a crucial role in the diffusion process and that their selective modification with carbon results in marked facilitation of subsurface hydrogen diffusion.

By virtue of these examples, we demonstrate how model studies on complex nanostructured materials may provide an atomistic view of processes at the gas-solid interface related to heterogeneous catalysis.

\section{INTRODUCTION}

Heterogeneous catalysts employed in technology are highly complex multicomponent materials, whose structural and chemical composition can be effectively tuned to enable high reaction rates and high selectivity. ${ }^{1}$ The complexity of these materials is their principal advantage that allows adjustment of chemical and adsorption properties of these materials to optimize their performance in a specific reaction. At the same time, this enormous chemical and structural complexity of real catalysts prevents detailed atomistic understanding of structure-reactivity relationships and hinders rational design of new catalytic materials. As a strategy to overcome this problem, we developed concepts to prepare model systems based on single crystalline oxide films, which are used as supports for metal and oxide nanoparticles. ${ }^{2}$ Such models allow us to introduce important complexity features inherent to realistic catalysts in a highly controlled way, while keeping them accessible for most of the surface science techniques available nowadays, and

Received: April 29, 2015

Published: September 14, 2015 

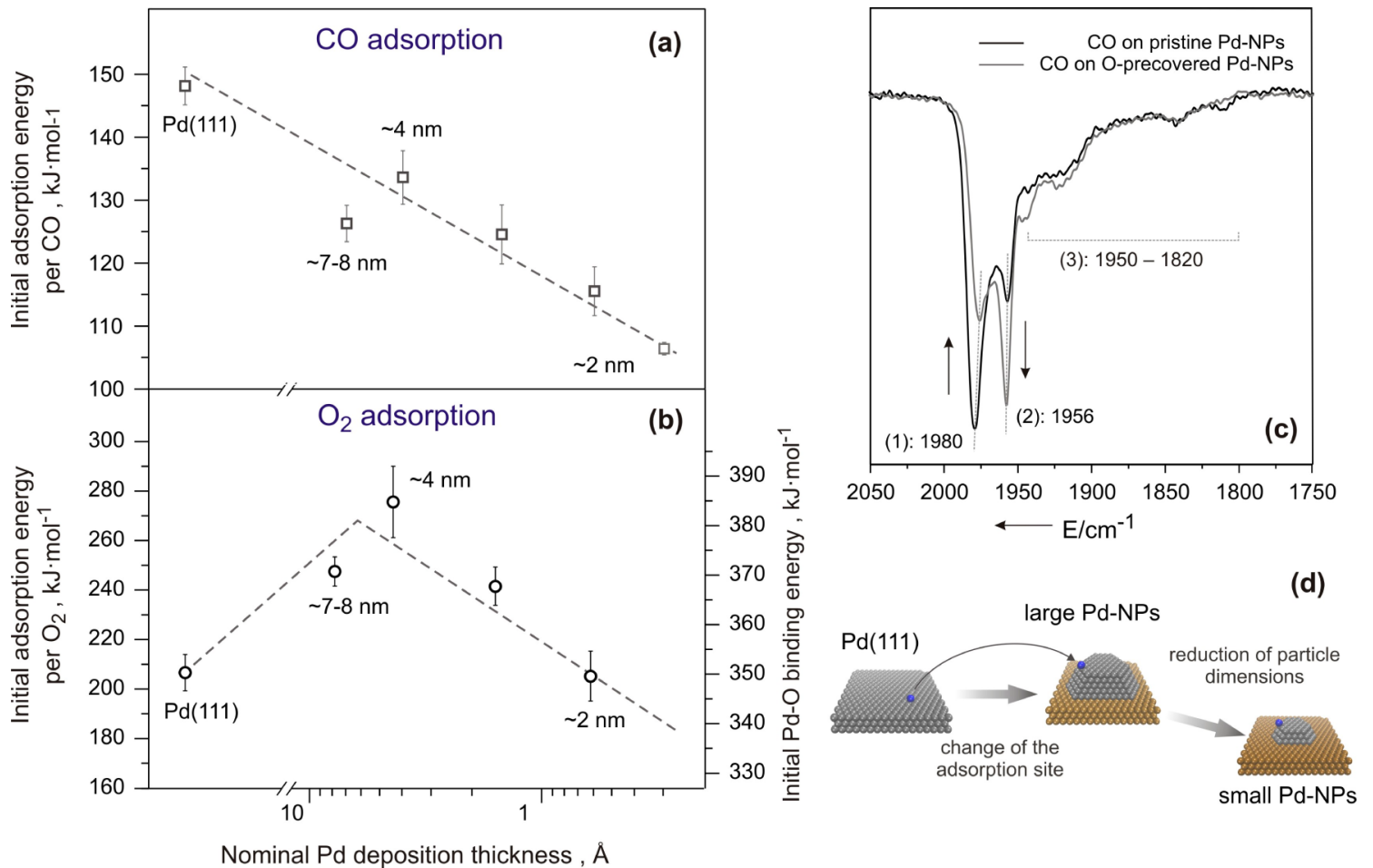

Figure 1. Initial adsorption energies for $\mathrm{CO}(\mathrm{a})$ and $\mathrm{O}_{2}(\mathrm{~b})$ molecules on $\mathrm{Pd} / \mathrm{Fe}_{3} \mathrm{O}_{4}(111) / \mathrm{Pt}(111)$ model catalysts and $\mathrm{Pd}(111)$ plotted as a function of the nominal thickness of the deposited Pd (from ref 25). The data are collected at $300 \mathrm{~K}$. (c) IRAS spectra for CO adsorbed on clean and O-precovered Pd nanoparticles (nominal deposition thickness $4 \AA$ ) supported on $\mathrm{Fe}_{3} \mathrm{O}_{4} / \mathrm{Pt}(111)$. (d) A model describing two microscopic trends affecting the binding energy: the local configuration of the adsorption site and the particle size. From ref 25, Copyright $2013 \mathrm{Wiley-VCH}$ Verlag $\mathrm{GmbH} \&$ Co. KGaA. Reproduced with permission.

systematically investigate the role of structural factors affecting the catalytic performance at the atomistic level.

For the controlled molecular design of new catalytic and other functional materials, a detailed knowledge of the reaction mechanisms, kinetics, and energetics of the adsorbate-surface interaction is required that provides a basis for fundamental understanding of how the surface binds the molecules and guides them through various elementary steps to the products. While a number of fundamental aspects of the elementary surface processes related to heterogeneous catalysis were understood on model single crystal surfaces, ${ }^{3}$ such fundamental information is not always available for practically important nanostructured materials. In the scope of the nanostructured model catalysts approach, we go beyond the traditional atomistic studies on single crystalline surfaces and try to answer the following question: how does the nanoscopic nature of metal particles affects their catalytic properties?

Here, we present two mechanistic case studies focusing on an atomistic-level understanding of elementary reaction steps in surface chemistry on metallic nanoparticles. Particularly, we investigate how the particle size and the flexibility of the atoms constituting metal clusters affect the binding energy of gasphase adsorbates by employing single crystal adsorption calorimetry. In the second study, we focus on hydrogen interaction with Pd nanoparticles and on the role of the surface modifiers, such as carbon, in this interaction. Specifically, we investigate diffusion of hydrogen into the volume of Pd clusters by carrying out an isotope exchange reaction and follow the changes in the hydrogen subsurface diffusion rate upon carbon deposition. By virtue of these examples, we demonstrate how model studies on complex model materials may help to unravel an atomistic view of processes at the solid-gas interface related to heterogeneous catalysis.

\section{BINDING ENERGY OF ADSORBATES: PARTICLE SIZE EFFECTS}

The performance of a heterogeneous catalyst strongly depends on the bond strength of the adsorbed surface species and on the relative thermodynamic stabilities of the reaction intermediates. It is one of the most important fundamental questions in catalysis research: How does the strength of a molecule-particle bond depend on the size of supported metal nanoparticle? Particularly, the interaction of carbon monoxide and oxygen with transition metal nanoparticles is of pivotal importance for a variety of industrially and environmentally relevant processes such as $\mathrm{CO}$ oxidation in exhaust catalytic converters and methane combustion.

The interaction of oxygen and carbon monoxide with palladium surfaces has been the subject of numerous studies, performed both on single crystal surfaces ${ }^{4-11}$ and on welldefined model systems consisting of Pd nanoclusters supported on thin oxide films. ${ }^{12-16}$ Presently, a very detailed microscopiclevel understanding of oxygen interaction with palladium is available, which turned out to be a complex interplay between chemisorption, ${ }^{5-7,10}$ diffusion of oxygen into the subsurface region and bulk, ${ }^{5,6,11}$ formation of surface oxide layers, ${ }^{8,17}$ refaceting, ${ }^{5,12}$ particle reconstruction, ${ }^{13}$ and bulk oxide formation. ${ }^{8,17}$ Despite this comprehensive understanding and general agreement on the surface chemistry of the oxygenpalladium system, quantitative information on binding energies of oxygen on Pd nanoparticles is still missing, which is precisely because of the richness of the surface chemistry as oxygen 
desorption occurs above the temperatures related to subsurface $\mathrm{O}$ diffusion, surface oxide formation, and particle restructuring. These side processes together with the restrictions imposed by the kinetic modeling of the temperature-programmed desorption (TPD) spectra strongly limit the quantitative determination of binding energies of oxygen on $\mathrm{Pd}$ nanoparticles by traditional desorption-based methods, which results in a strong scatter of data available in literature. The energetics of $\mathrm{CO}$ interaction with well-defined metal nanoparticles were previously addressed indirectly in nonisothermal TPD experiments ${ }^{18}$ and in isothermal modulated molecular beam studies. ${ }^{12}$ However, these indirect methods based on modeling of the desorption process did not provide a clear trend in the changes of the adsorption energy with the particle size: whereas the TPD studies found a decrease of the adsorption energy by about $10 \mathrm{~kJ} \cdot \mathrm{mol}^{-1}$ on the $2.5 \mathrm{~nm}$ sized Pd particles compared with that of the extended single crystal surfaces, the kinetic model used for analysis of the molecular beam experiments predicted a pronounced increase of the adsorption energy by about $35 \mathrm{~kJ} \cdot \mathrm{mol}^{-1}$ on the particles smaller than $1.5 \mathrm{~nm}$. A strategy to overcome the shortcomings of the desorption-based methods is a direct calorimetric measurement of adsorption enthalpies under isothermal conditions. ${ }^{19}$ Here, we report on the first direct calorimetric measurement of oxygen and $\mathrm{CO}$ binding energies on $\mathrm{Pd}$ nanoparticles investigated as a function of particle size and with the reference to a $\mathrm{Pd}(111)$ single crystal. We apply a newly developed UHV single crystal adsorption calorimeter (SCAC) ${ }^{20}$ based on molecular beam techniques in combination with infrared reflection adsorption spectroscopy (IRAS) to investigate the effect that the reduced dimensionality of metallic particles has on the interaction strength with adsorbates.

The model surfaces employed in this study consist of $\mathrm{Pd}$ nanoparticles supported on a well-ordered thin $\mathrm{Fe}_{3} \mathrm{O}_{4}$ film grown on a $\mathrm{Pt}(111)$ single crystal (for details of the preparation procedure and structural characterization by scanning tunneling microscopy (STM) see ref 16). Five different supported systems with different particle sizes were prepared with the average number of $\mathrm{Pd}$ atoms per particle of about 110, 220, 430,720 , and 4760 (1.8-8 $\mathrm{nm}$ in diameter). According to STM data, the particles are crystalline and grow predominantly in (111) orientation with a small fraction of (100) facets. All Pd clusters are three-dimensional, as evidenced by the comparison of the average number of $\mathrm{Pd}$ atoms per particle and the "footprint" of the clusters obtained by STM as well as by transmission electron microscopy (TEM). This observation excludes the possibility of strong metal-support interaction in this system. By applying IRAS, TPD, and sticking coefficient measurements, we proved that $\mathrm{CO}$ does not adsorb at the $\mathrm{Fe}_{3} \mathrm{O}_{4}$ support at the investigated adsorption temperature of $300 \mathrm{~K}$. The $\mathrm{Fe}_{3} \mathrm{O}_{4}$ film was chosen as a substrate exhibiting an outstanding stability under applied experimental conditions.

The dependence of initial $\mathrm{CO}$ adsorption energies (Figure 1a), corresponding to adsorption of a few $\mathrm{CO}$ molecules per $\mathrm{Pd}$ nanoparticle, on particle size shows a pronounced trend: the initial heat of adsorption decreases with decreasing particle size, from $126 \pm 7 \mathrm{~kJ} \cdot \mathrm{mol}^{-1}$ on $8 \mathrm{~nm} \mathrm{Pd}$ particles to $106 \pm 2 \mathrm{~kJ}$. $\mathrm{mol}^{-1}$ on $1.8 \mathrm{~nm}$ clusters. Additionally, all investigated particles showed smaller initial adsorption enthalpy compared with the single crystal surface $\left(149 \mathrm{~kJ} \cdot \mathrm{mol}^{-1}\right)$. With this, it was clearly shown for the first time by a direct calorimetric method that the initial adsorption energy of $\mathrm{CO}$ strongly decreases with decreasing particle size even despite the fact that the relative fraction of the low-coordinated adsorption sites (edges and corners), which would normally be expected to bind the adsorbates more strongly, increases on small particles. Obviously, some other size-dependent properties of a $\mathrm{Pd}$ nanocluster must be responsible for the observed decrease of the initial $\mathrm{CO}$ adsorption energy with decreasing particle size.

Two alternative microscopic effects may contribute to the observed decrease of the initial $\mathrm{CO}$ adsorption heat with decreasing particle size: (i) weakening of the chemisorptive interaction and (ii) reduction of the van-der-Waals attraction. First, the decrease of the chemisorption energy can result from the contraction of the lattice parameter of a small metal nanoparticle, which was demonstrated experimentally ${ }^{21}$ and predicted theoretically. ${ }^{22}$ This phenomenon has been rationalized to result from the decreasing average coordination number of atoms with higher surface-to-volume ratio of the cluster. This lattice contraction in small metal clusters was theoretically shown to result in a reduction of the adsorbate binding energy. ${ }^{23}$ This finding agrees with the principle of bond order conservation: ${ }^{24}$ in "contracted" clusters, one expects weaker adsorption bonds and stronger binding within the adsorbate as a result of saturated valences among the substrate atoms. A second reason for adsorption energy of a gas-phase molecule to decrease on the small metal clusters is a feasible weakening of dispersion force (van-der-Waals interaction) that is induced by the dynamic response of bulk electrons of the metal to charge density fluctuations in an adsorbed molecule. The large overall magnitude of the decrease in the heat of adsorption obtained in our study (about $40 \mathrm{~kJ} \cdot \mathrm{mol}^{-1}$ relative to the $\mathrm{Pd}(111)$ surface) allows us to suggest that most likely reduction of both van-derWaals interaction and chemisorption strength contributes to the overall decrease of the adsorption heat.

To address the dependence of the initial $\mathrm{O}$ adsorption energies on Pd particle size, four different supported model systems with different nominal Pd deposition thicknesses ranging from 0.6 to $7 \AA$ (particle size $2-8 \mathrm{~nm}$ ) were investigated by SCAC. By applying TPD and sticking coefficient measurements, we proved that $\mathrm{O}_{2}$ does not adsorb at the $\mathrm{Fe}_{3} \mathrm{O}_{4}$ support at the investigated adsorption temperature of $300 \mathrm{~K}$. The initial heats of adsorption on all supported systems are displayed in Figure $1 \mathrm{~b}$ as a function of the nominal Pd deposition thickness together with data measured on $\mathrm{Pd}(111)$. The energy value of $205 \mathrm{~kJ} \cdot \mathrm{mol}^{-1}$ obtained for $\mathrm{O}_{2}$ adsorption on $\mathrm{Pd}(111)$ in the lowest coverage limit is in a good agreement with literature values of $\mathrm{O}$ adsorption energies at low coverages situated in the range of 210 to $230 \mathrm{~kJ} \cdot \mathrm{mol}^{-1}$. $5,7,9$ In contrast, an unexpectedly high $\mathrm{O}_{2}$ adsorption high energy value $\left(275 \mathrm{~kJ} \cdot \mathrm{mol}^{-1}\right)$ was measured on the largest $\mathrm{Pd}$ nanoparticles. This value cannot be compared with any value reported in previous TPD studies, neither for low Miller index Pd surfaces nor for stepped Pd crystals, which typically span the range between 190 and $230 \mathrm{~kJ} \cdot \mathrm{mol}^{-1} \cdot 4,6,7,11$ Thus, this high initial adsorption energy must be related to some structural feature inherent to $\mathrm{Pd}$ nanoparticles that cannot be reproduced even by the steps of the high Miller index Pd surfaces. Such adsorption sites on $\mathrm{Pd}$ nanoparticles might be the low coordinated surface sites (edges, corners). In previous STM studies on supported Pd nanoparticles, oxygen was shown to modify such sites first, indicating that they are the strongest adsorption sites on a Pd cluster. ${ }^{15}$

To check the feasibility of this explanation, we carried out $\mathrm{CO}$ titration experiments to identify the preferential adsorption sites of oxygen on Pd nanoparticles. Based on the IR spectra 
shown in Figure 1c, it was possible to determine that adsorbed oxygen preferentially accumulates at the low-coordinated sites (particle edges and corners) in the low-coverage limit. For a detailed discussion of IRAS data, see ref 25 . Briefly, the spectrum of $\mathrm{CO}$ on pristine Pd nanoparticles is dominated by a sharp adsorption feature at $1980 \mathrm{~cm}^{-1}$ (1) that mainly originates from bridge-bonded $\mathrm{CO}$ adsorbed at low coordinated surface sites, such as particle edges and corners. Additionally, a peak of lower intensity at $1956 \mathrm{~cm}^{-1}$ (2) is observed alongside a broad low-frequency shoulder (3; 1950$\left.1820 \mathrm{~cm}^{-1}\right)$. These latter bands (2 and 3 ) were previously assigned to $\mathrm{CO}$ adsorbed on bridge and hollow sites predominantly on $\mathrm{Pd}(111)$. After adsorption of submonolayer amounts of oxygen, drastic changes are observed in the spectrum for the adsorbed CO. The edge-related peak at 1980 $\mathrm{cm}^{-1}$ (1) is strongly attenuated, whereas the feature at 1956 $\mathrm{cm}^{-1}$ gains in intensity. Taking into account these observations and the dipole coupling effects, we conclude that oxygen preferentially accumulates at the low-coordinated sites such as particle edges and corners. Therefore, we can unambiguously attribute the unexpectedly high initial adsorption energy (275 $\left.\mathrm{kJ} \cdot \mathrm{mol}^{-1}\right)$, measured in a calorimetric experiment on supported Pd nanoparticles, to oxygen adsorption at edge and corner sites.

The dependence of the $\mathrm{O}$ adsorption energy on the particle size shows a clear trend: it strongly rises from $\sim 205$ to $250-$ $275 \mathrm{~kJ} \cdot \mathrm{mol}^{-1}$ when changing from $\mathrm{Pd}(111)$, where oxygen adsorbs at 3-fold-hollow sites, to large Pd nanoclusters, where oxygen first occupies particles edges. However, if the local adsorption site (the edge/corner site) was preserved, and only the particle size was reduced, the initial oxygen adsorption energy was observed to strongly decrease, reaching the value of $205 \mathrm{~kJ} \cdot \mathrm{mol}^{-1}$ on the smallest Pd nanoparticles. Thus, the reduction of the Pd cluster size results in an opposite trenddecrease of the adsorption energy, with a magnitude that is comparable to the effect of the change of the local adsorption environment. The latter trend, decreasing adsorption energy with decreasing particle size, ${ }^{26}$ coincides with the trend observed for $\mathrm{CO}$ adsorption.

Strong decrease of the oxygen binding energy on the particles with decreasing particle size can be rationalized within the same microscopic picture that was discussed earlier for $\mathrm{CO}$ adsorption: weakening of the chemisorptive interaction due to reduction of $\mathrm{Pd}-\mathrm{Pd}$ interatomic bond length in contracting clusters and reduction of the van-der-Waals attraction. The latter effect is expected to play a more important role for the interaction of $\mathrm{CO}$ with Pd clusters and be of minor importance in case of oxygen.

In summary, we investigated, for the first time, the particle size dependence of $\mathrm{CO}$ and $\mathrm{O}_{2}$ adsorption energy on welldefined Pd nanoparticles and on an extended $\mathrm{Pd}(111)$ single crystal surface in a direct calorimetric study. Two microscopic structural parameters were identified to affect the binding strength of the adsorbates in two principle ways: (i) via the change of the local adsorption environment that can result, for example, in stronger adsorbate bonding at the particle edges and (ii) via contraction of Pd lattice in small clusters and a concomitant weakening of chemisorptive interaction. The change of the local adsorption environment from a 3-fold hollow position on extended $\operatorname{Pd}(111)$ single crystal to an edge site of Pd nanoparticles results in a strong increase of oxygen binding energy by about $70 \mathrm{~kJ} \cdot \mathrm{mol}^{-1}$. The unexpectedly strong binding energy of oxygen at the particles edges exceeds all literature values of oxygen adsorption energies at stepped single crystal surfaces. On the other hand, if the local configuration of the adsorption site is kept constant (O adsorption at the edges/ corners of Pd nanoparticles), the reduction of the cluster size leads to a pronounced decrease of oxygen binding energy from $275 \mathrm{~kJ} \cdot \mathrm{mol}^{-1}$ observed on the large clusters to $205 \mathrm{~kJ} \cdot \mathrm{mol}^{-1}$ measured for the smallest investigated nanoparticles. In total, these effects were found to result in counteracting trends: the increase of the $\mathrm{O}-\mathrm{Pd}$ binding strength due to adsorption at the low-coordinated surface sites and the decrease of the $\mathrm{O}-\mathrm{Pd}$ adsorption energy due to reduced particle dimensions.

For $\mathrm{CO}$ adsorption, the local coordination of the binding site was observed not to play a major role in determining the binding energy, while the reduced particle size was found to result in strong decrease of the $\mathrm{CO}$ adsorption energy. This latter trend coincides with the particle size dependence of $\mathrm{O}$ adsorption energy on Pd particles.

In total, the decreasing adsorption energy observed in our studies with decreasing particle size both for $\mathrm{CO}$ and oxygen adsorption is indicative of the general nature of this phenomenon, which might be connected to the theoretically predicted weakening of chemisorptive interaction due to the contraction of the lattice parameter of the $\mathrm{Pd}$ cluster.

\section{SUBSURFACE HYDROGEN DIFFUSION INTO PD NANOPARTICLES: ROLE OF LOW-COORDINATED SURFACE SITES AND FACILITATION BY CARBON}

The ability of the nanosized clusters of transition metals to absorb atomic hydrogen has pivotal importance for a wide variety of the industrially relevant applications, including heterogeneous catalysis. Recently, the crucial role of hydrogen absorbed in the subsurface region of the active metal phase was established in a number of surface science studies on hydrogenation of alkenes and alkynes. ${ }^{27-29}$ For these applications, activated hydrogen diffusion into the subsurface of a metal particle is an essential elementary step, which can critically control the overall performance of the catalyst. ${ }^{29,30}$ Recent mechanistic studies on olefin hydrogenation evidence that truly catalytic sustained reactivity on Pd nanoparticles can be achieved only if the subsurface hydrogen reservoir can be effectively replenished under reaction conditions and propose that this process can efficiently proceed only in the presence of carbon. $^{29,30}$ Even though the important role of carbonaceous deposits for the activity and selectivity of transition metals has been recognized for a long time in the catalytic community, the role of how carbon acts in surface processes involving hydrogen at the microscopic level is not yet understood.

The interaction of hydrogen with transition metals was extensively studied by both experimental and theoretical methods for several decades, ${ }^{31-34}$ but the microscopic details of the diffusion process into the subsurface region remain largely unexplored. This is mainly because the range of the experimental tools sensitive to hydrogen and capable of addressing the dynamics of hydrogen diffusion in a timeresolved way are very limited.

In this study, we experimentally addressed the kinetics of hydrogen diffusion into the subsurface on well-defined model $\mathrm{Pd}$ nanoparticles supported on a well-ordered $\mathrm{Fe}_{3} \mathrm{O}_{4} / \mathrm{Pt}(111)$ thin film and on $\mathrm{Pd}(111)$ by performing pulsed multimolecular beam experiments. Particular emphasis was placed on understanding the role of atomically flexible low-coordinated surface sites, such as edges and corners of Pd nanoparticles, on the diffusion process and on exploring how modification of these sites with carbon affects the subsurface diffusion rate. To probe 
hydrogen subsurface diffusion, the $\mathrm{H}_{2}+\mathrm{D}_{2} \rightarrow \mathrm{HD}$ exchange reaction was conducted in the temperature regime where this reaction is limited by the formation rate of subsurface $H(D)$ species.

The model catalyst consists of $\mathrm{Pd}$ nanoparticles of $\sim 7 \mathrm{~nm}$ size that are mainly terminated by (111) facets and exhibit lowcoordinated surface Pd atoms incorporated into edges, corners, and (100) facets. ${ }^{16}$ Submonolayer amounts of carbon were deposited at the particle by thermal decomposition of butene, ${ }^{29}$ and it was established spectroscopically that carbon occupies predominately edge sites of the Pd nanoparticles while leaving the regular (111) terraces nearly unchanged. ${ }^{35}$ Recent theoretical calculations suggest enhanced stability of carbon between the first and the second Pd layer. ${ }^{36}$

Figure 2a shows a desorption trace of $\mathrm{D}_{2}$ from $\mathrm{Pd}$ nanoparticles. Two distinct peaks with maxima at 295 and

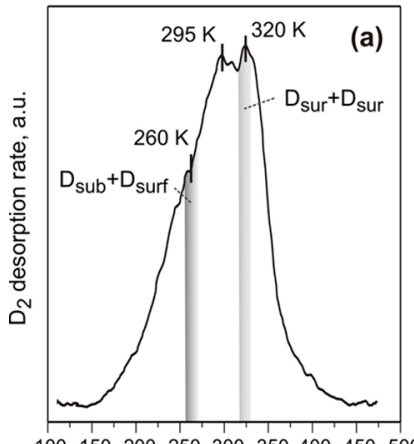

100150200250300350400450500 Temperature, $\mathrm{K}$ $\operatorname{Pd}(111)$
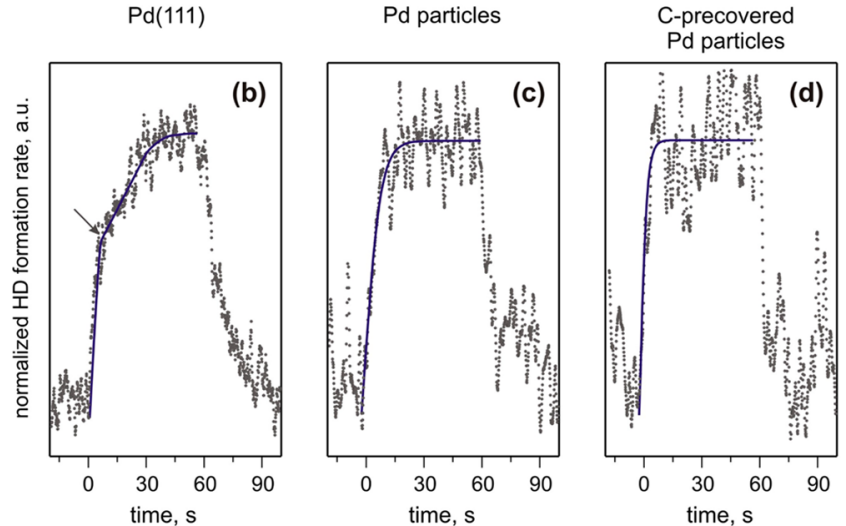

Figure 2. (a) TPD spectrum obtained after adsorption of $3.1 \mathrm{~L}$ of $\mathrm{D}_{2}$ on $\mathrm{Pd} / \mathrm{Fe}_{3} \mathrm{O}_{4} / \mathrm{Pt}(111)$ model catalyst at $100 \mathrm{~K}$. Time evolution of the $\mathrm{HD}$ formation rate obtained at $280 \mathrm{~K}$ on (b) $\mathrm{Pd}(111)$, (c) pristine $\mathrm{Pd}$ nanoparticles supported on $\mathrm{Fe}_{3} \mathrm{O}_{4} / \mathrm{Pt}(111)$, and (d) C-precovered $\mathrm{Pd}$ nanoparticles. (e) The steady state $\mathrm{HD}$ formation rates obtained on the pristine and $\mathrm{C}$-precovered $\mathrm{Pd}$ nanoparticles supported on $\mathrm{Fe}_{3} \mathrm{O}_{4}$ / $\mathrm{Pt}(111)$ at 260 and $320 \mathrm{~K}$ at a constant $\mathrm{H}_{2}: \mathrm{D}_{2}$ ratio. Reproduced from ref 37. Copyright 2012 American Chemical Society.

$320 \mathrm{~K}$ can be identified. Previously, by performing hydrogen depth profiling via resonant nuclear reaction analysis (rNRA), ${ }^{34}$ it was possible to clearly attribute the high temperature peak to associative recombination of strongly bound $\mathrm{H}(\mathrm{D})$ species adsorbed on the surface, which will be indicated in the following as $\mathrm{H}_{\text {surf }}\left(\mathrm{D}_{\text {surf }}\right)$. The desorption of $\mathrm{H}_{2}\left(\mathrm{D}_{2}\right)$ in the low temperature regime (between 200 and $\sim 300 \mathrm{~K}$ ) was found to be associated with the depletion of weakly bound $\mathrm{H}(\mathrm{D})$ species absorbed in the bulk of $\mathrm{Pd}$ particles, further indicated as
$\mathrm{H}_{\text {sub }}\left(\mathrm{D}_{\text {sub }}\right)$. It should be emphasized that depletion of only subsurface species, $\mathrm{H}_{\text {sub }}\left(\mathrm{D}_{\text {sub }}\right)$, was observed upon heating in this temperature range by rNRA, while the concentration of surface $H_{\text {surf }}\left(D_{\text {surf }}\right)$ remained constant, implying that the lowtemperature desorption pathway involves consumption of at least one subsurface $\mathrm{H}_{\text {sub }}\left(\mathrm{D}_{\text {sub }}\right)$ species. It is likely that a surface-adsorbed $\mathrm{H}_{\text {surf }}\left(\mathrm{D}_{\text {surf }}\right)$ atom can also be involved into this low-temperature pathway as a participant. In this case, a desorption event must be accompanied by a simultaneous replenishment of the surface $\mathrm{H}_{\text {surf }}\left(\mathrm{D}_{\text {surf }}\right)$ reservoir from the $\mathrm{H}_{\text {sub }}\left(\mathrm{D}_{\text {sub }}\right)$ reservoir.

The clear distinction of two desorption regimes allowed us to separately probe the kinetics of the processes dominated by either (i) associative desorption of subsurface species, $\mathrm{H}_{\text {sub }}\left(\mathrm{D}_{\text {sub }}\right)$, or (ii) processes governed by desorption of more strongly bound surface species, $\mathrm{H}_{\text {surf }}\left(\mathrm{D}_{\text {surf }}\right)$. For this purpose, the following experimental approach was used: we exposed the model surface to a continuous high flux $\mathrm{D}_{2}$ molecular beam until both the surface and the volume of $\mathrm{Pd}$ particles were saturated with $\mathrm{D}$ and then a second molecular beam applied 60 s long pulses of low flux $\mathrm{H}_{2}$ with a reactant of ratio $\mathrm{D}_{2} / \mathrm{H}_{2}=71$ upon the surface. The time evolution of the reaction product $\mathrm{HD}$ was monitored by quadrupole mass spectrometry. At a sufficiently low reaction temperature $(260-280 \mathrm{~K})$, the $\mathrm{HD}$ formation rate is dominated by association of subsurface-related species (note that this pathway results in the consumption of the subsurface species but does not necessarily occur via recombination of two subsurface $\mathrm{H}$ atoms, see detailed discussion in ref 37 ).

Figure $2 \mathrm{~b}-\mathrm{d}$ shows the time evolution of the HD formation rate in response to the $\mathrm{H}_{2}$ beam pulse in the low-temperature regime for three surfaces: $\operatorname{Pd}(111)$ and clean and C-precovered Pd nanoparticles. On $\operatorname{Pd}(111)$ (Figure 2b), a clear parallel reaction behavior can be observed: after the $\mathrm{H}_{2}$ beam was opened, the rate of $\mathrm{HD}$ formation increases rapidly within approximately $4 \mathrm{~s}$ and then exhibits an inflection point before reaching a steady state value after $\sim 40 \mathrm{~s}$. The height of the final plateau is about twice that of the inflection point. On clean and C-precovered Pd particles (Figure 2c,d), a similar fast increase of the reaction rate is observed initially to the inflection point (within the first $4 \mathrm{~s}$ ), but the following increase to a steady state level occurs more rapidly, in about $9 \mathrm{~s}$ for the $\mathrm{C}$-free and $6 \mathrm{~s}$ for the C-precovered Pd particles.

The initial fast-rising component of the HD formation rate can be rationalized in terms of the known desorption behavior of hydrogen from Pd by HD formed in the reaction between $\mathrm{D}_{\text {sub }}$, which is already present in the saturated particles, and surface $\mathrm{H}_{\text {surf }}$ which is very quickly formed by spontaneous nonactivated dissociation of $\mathrm{H}_{2}$ in the pulsed beam, typical for a number of the transition metal surfaces including $\mathrm{Pd}^{31}$ In contrast, the slower component of the HD rate can originate from reaction between $\mathrm{D}_{\text {surf }}$ and $\mathrm{H}_{\text {sub }}$, the latter species being formed in a slow activated process of $\mathrm{H}$ diffusion into the subsurface. ${ }^{31}$ Within the scope of this model, the reaction rate, $R_{\mathrm{HD}}$, of the low-temperature channel includes two terms:

$$
R_{\mathrm{HD}}(t)=k_{\text {sub }}\left[D_{\text {sub }}\right]\left[H_{\text {surf }}(t)\right]+k_{\text {sub }}\left[D_{\text {surf }}\right]\left[H_{\text {sub }}(t)\right]
$$

with $k_{\text {sub }}$ being the reaction constant between one surface and one subsurface $\mathrm{H}(\mathrm{D})$ atom. For more details of the kinetic model and a comprehensive discussion, see ref 37 . The first term in this equation describes the fast rise of the $\mathrm{HD}$ formation rate within the first $4 \mathrm{~s}$ due to association of a quickly formed $\mathrm{H}_{\text {surf }}$ and already available $\mathrm{D}_{\text {sub }}$, while the second term is 
related to the association process between already present $D_{\text {surf }}$ and slowly formed $\mathrm{H}_{\text {sub}}$.

It should be pointed out that the observed parallel reaction behavior, consisting of two clear reaction branches with two different characteristic times, suggests a reaction mechanism involving the direct association of one subsurface $\mathrm{D}_{\text {sub }}\left(\mathrm{H}_{\text {sub }}\right)$ with one surface-adsorbed $\mathrm{H}_{\text {surf }}\left(\mathrm{D}_{\text {surf }}\right)$ species. In contrast, if only one type of hydrogen species, formed with the same time constant, were involved in HD formation, only a single reaction channel could be observed.

The initial formation rate of $\mathrm{H}_{\text {sub }}$ directly depends on the rate constant for subsurface hydrogen diffusion. The slow rise of the second branch of the HD formation reaction to the steady state on the close-packed $\mathrm{Pd}(111)$ indicates a slow formation rate of $\mathrm{H}_{\text {sub }}$ on the extended single crystal and, hence, low subsurface diffusion rate constant, $k_{\text {diff. }}$ Faster $\mathrm{HD}$ formation rate in the second branch and, consequently, faster formation of $\mathrm{H}_{\text {sub }}$ on Pd particles can be attributed to much faster diffusion rate constant, $k_{\text {diff, }}$ on the particles. This might be a result of the atomic flexibility of the nanoclusters, particularly at the lowcoordinated surface sites such as edges and corners, which allows easier $\mathrm{H}$ penetration into the volume of the particles. Employing detailed kinetic modeling of the experimental data, ${ }^{37}$ it was possible to calculate that the diffusion rate constant, $k_{\text {diff, }}$ of $\mathrm{H}$ from the surface into the subsurface region of Pd is faster on clean Pd nanoparticles by a factor of $\sim 5.4$ compared with that on $\operatorname{Pd}(111)$. What is more important, Cdeposition at the edges of $\mathrm{Pd}$ nanoclusters was found to increase the diffusion rate constant, $k_{\text {diff, }}$ by a factor of roughly two compared with clean Pd nanoparticles.

Finally, we probed the HD exchange in a broad range of pressure conditions in steady state regime. Figure $2 \mathrm{e}$ shows the steady state $\mathrm{HD}$ formation rates obtained in these experiments for the reaction temperatures 260 and $320 \mathrm{~K}$. Carbon deposition was found to affect the HD formation rate in a dramatically different way for the two reaction temperatures: whereas at $320 \mathrm{~K}$, preadsorbed carbon reduces the overall reaction rate by about $30 \%$, the reaction rate increases by about $100 \%$ at $260 \mathrm{~K}$ on the carbon-precovered particles for all pressures studied. The decreased $\mathrm{HD}$ formation rate in the high-temperature regime $(320 \mathrm{~K})$, where $\mathrm{HD}$ formation proceeds via recombination of the surface-adsorbed species, $\mathrm{H}_{\text {surf }}$ and $\mathrm{D}_{\text {surf }}$, can be easily rationalized as a consequence of blocking of adsorption sites by carbon. Interestingly, even though a part of the surface is blocked by carbon, the HD formation rate significantly increases in the low-temperature regime $(260 \mathrm{~K})$, where desorption is dominated by recombination of the subsurface-related species $\left(\mathrm{H}_{\text {surf }}+\mathrm{D}_{\text {sub }}\right.$ and $\left.\mathrm{D}_{\text {surf }}+\mathrm{H}_{\text {sub }}\right)$. This effect can be explained only by the higher formation rate of the subsurface $\mathrm{H}_{\text {sub }}\left(\mathrm{D}_{\text {sub }}\right)$ species on the $\mathrm{C}$-modified particles resulting in a higher steady state concentration by a factor of 2 of these species in the subsurface (note that at this low temperature, the surface is saturated with $\mathrm{H}_{\text {surf }}$ and $\mathrm{D}_{\text {surf }}$ so that the total coverage of $\mathrm{H}_{\text {surf }}+\mathrm{D}_{\text {surf }}$ is equal to one for all surfaces). This result is in perfect agreement with the time evolution of the HD product presented in Figure $2 b-$ $d$, which also suggests that the effective subsurface diffusion rate constant $\left(k_{\text {diff }}\right)$ on the Pd particles increases after $\mathrm{C}$ deposition by about factor of 2 . It should be noted that at the microscopic level, facilitation of the subsurface $\mathrm{H}$ diffusion through the $\mathrm{C}$ modified low-coordinated sites is most likely even more pronounced. Indeed, the nearly $100 \%$ increase of the overall subsurface diffusion rate arises from modification of only 20\% of the surface sites constituting edges and corners of $\mathrm{Pd}$ particles. Microscopically, this means that the local diffusion rate through these $\mathrm{C}$-modified sites increases by at least an order of magnitude. These results are in the excellent qualitative and in a good quantitative agreement with the theoretical predictions. ${ }^{38}$ These theoretical calculations revealed that carbon deposition at the particles edges results in expansion of Pd lattice with vanishing of the activation barrier for hydrogen subsurface diffusion. Such effect could be observed only at the atomically flexible Pd nanoparticles but not at the atomically stiff extended $\operatorname{Pd}(111)$ surface, which demonstrates conceptual importance of the atomic flexibility of metal nanoparticles for hydrogen diffusion into subsurface.

In conclusion, we have probed the kinetics of $\mathrm{H}$ subsurface diffusion in Pd nanoparticles using pulsed multimolecular beam experiments. The HD exchange reaction was carried out in a temperature regime at which it is limited by the formation of subsurface $\mathrm{H}_{\text {sub }}$ species, and the process of subsurface $\mathrm{H}$ diffusion occurs at experimentally measurable time scales. By comparing the time evolution of the HD product on the model supported $\mathrm{Pd}$ nanoparticles and extended $\mathrm{Pd}(111)$ surface, we show that the $\mathrm{H}$ subsurface diffusion critically depends on the structure of the surface and is considerably facilitated on the atomically flexible $\mathrm{Pd}$ nanoparticles. Moreover, in agreement with theoretical predictions, modification of the atomically flexible low-coordinated surface sites, such as edges and corners, with carbon was found to substantially increase the subsurface diffusion rate. The observed phenomena provide important insights into the microscopic details of hydrogen interaction with technically relevant transition metal surfaces and underline the exceptional importance of the atomic flexibility of metal nanoparticles and the surface modifiers that are usually formed under catalytic reaction conditions.

\section{CONCLUDING REMARKS}

Two case studies presented in this Account were supposed to demonstrate and underline how model studies on nanostructured surfaces may be employed to investigate complex problems in catalysis and provide fundamental insight into surface processes on technologically relevant surfaces. In the first study, we have shown that the reduced dimensions of the Pd substrate can affect the binding strength of the adsorbates in two principle ways: (i) via the change of the local adsorption environment that can result, for example, in stronger adsorbate bonding at the particle edges and (ii) via contraction of $\mathrm{Pd}$ lattice in small clusters and a concomitant weakening of chemisorptive interaction. With this, particle size effects can strongly affect the equilibrium coverage of the surface species and the overall reactivity. The presented experimental data provide important benchmarks for upcoming theoretical calculation on materials with reduced dimensions. In the second case study, we discussed interaction of hydrogen with $\mathrm{Pd}$, particularly, diffusion of hydrogen into the volume of $\mathrm{Pd}$ material, a technologically very important process. We were able to demonstrate that, in line with theoretical predictions, carbon deposited at the low-coordinated sites (edges, corners) of $\mathrm{Pd}$ nanoclusters strongly facilitates hydrogen penetration into subsurface and allows it to maintain high concentrations of subsurface hydrogen under the technologically relevant reaction conditions. Here, the atomic flexibility of small Pd nanoparticles and their ability to expand the lattice in the presence of carbon was theoretically predicted to be responsible for facilitation of subsurface hydrogen diffusion. Our experimental 
data confirm this hypothesis and provide important atomistic insights into the surface chemistry involving hydrogen. These findings illustrate a very complex interplay between the inherent structural properties of metallic nanoparticles, such as size and the atomic flexibility, and the effects of surface modifiers. We believe that these mechanistic insights into surface chemistry will help to close a gap between models and real catalysts.

\section{ASSOCIATED CONTENT}

\section{S Supporting Information}

The Supporting Information is available free of charge on the ACS Publications website at DOI: 10.1021/acs.accounts.5b00237.

Preparation and characterization of $\mathrm{Pd}$ nanoparticles (PDF)

\section{AUTHOR INFORMATION}

\section{Corresponding Author}

*E-mail: schauermann@fhi-berlin.mpg.de.

Notes

The authors declare no competing financial interest.

\section{Biographies}

Swetlana Schauermann studied chemistry and received her Ph.D. in 2005 in the group of H.-J. Freund investigating the catalytic activity of model supported nanoparticles by molecular beam techniques and vibrational spectroscopy. Between 2006 and 2007, she worked as a postdoctoral fellow with R. M. Lambert (Cambridge University) and C. T. Campbell (University of Washington) and later became a group leader in Fritz-Haber-Institut. Since 2015, she is Associate Professor at Kiel University. Her current research is focused on kinetics of complex surface reactions and on thermodynamics of gas-surface interactions.

Hans-Joachim Freund studied physics and chemistry at the University of Cologne where he received his Ph.D. in 1978 and his habilitation in 1983. Between 1979 and 1981, he worked in the Physics Department at the University of Pennsylvania as a postdoctoral fellow. In 1983, he became Associate Professor at Erlangen University and in 1987 Professor at Bochum University, and in 1995, he accepted a position as scientific member and director of the Department of Chemical Physics at the Fritz-Haber-Institut der Max-Planck-Gesellschaft in Berlin. He serves as Honorary Professor of five universities.

\section{ACKNOWLEDGMENTS}

We are indebted to M. Wilde (Tokyo), K. Fukutani (Tokyo), K. Neyman (Barcelona), and N. Rösch (Munich), as well as their groups for fruitful collaborations. We thank our collaborators mentioned in the references. The authors also acknowledge support from the Cluster of Excellence UNICAT granted by the German Science Foundation (DFG) as well as by the Verband der Chemischen Industrie (VCI).

\section{REFERENCES}

(1) Ertl, G.; Knözinger, H.; Weitkamp, J. Handbook of Heterogeneous Catalysis; VCH: Weinheim, Germany, 1997.

(2) Bäumer, M.; Freund, H.-J. Metal deposits on well-ordered oxide films. Prog. Surf. Sci. 1999, 61, 127-198.

(3) Ertl, G. Basic Principles; Reactions at Solid Surfaces; John Wiley \& Sons, Inc.: Hoboken, NJ, 2010; pp 1-19.

(4) Zheng, G.; Altman, E. I. The oxidation mechanism of $\mathrm{Pd}(100)$. Surf. Sci. 2002, 504, 253-270.
(5) Zheng, G.; Altman, E. I. The oxidation of $\operatorname{Pd}(111)$. Surf. Sci. 2000, 462, 151-168.

(6) Li, F.; Allegretti, F.; Surnev, S.; Netzer, F. P.; Zhang, Y.; Zhang, W. B.; Reuter, K. Oxygen adsorption on stepped $\mathrm{Pd}(100)$ surfaces. Surf. Sci. 2010, 604, 1813-1819.

(7) Klötzer, B.; Hayek, K.; Konvicka, C.; Lundgren, E.; Varga, P. Oxygen-induced surface phase transformation of $\mathrm{Pd}(111)$ : sticking, adsorption and desorption kinetics. Surf. Sci. 2001, 482-485 (Part 1), 237-242.

(8) Engel, T. A molecular beam investigation of $\mathrm{He}, \mathrm{CO}$, and $\mathrm{O} 2$ scattering from $\operatorname{Pd}(111)$. J. Chem. Phys. 1978, 69, 373-385.

(9) Conrad, H.; Ertl, G.; Küppers, J.; Latta, E. E. Interaction of NO and $\mathrm{O} 2$ with $\mathrm{Pd}(111)$ surfaces. II. Surf. Sci. 1977, 65, 245-260.

(10) Guo, X.; Hoffman, A.; Yates, J. T. Adsorption kinetics and isotopic equilibration of oxygen adsorbed on the $\mathrm{Pd}(111)$ surface. $J$. Chem. Phys. 1989, 90, 5787-5792.

(11) Davies, P. W.; Lambert, R. M. Structural stability and chemisorption properties of a stepped palladium surface: $\mathrm{O} 2$ and no on $\operatorname{Pd}(331)$. Surf. Sci. 1981, 110, 227-249.

(12) Henry, C. R. Surface studies of supported model catalysts. Surf. Sci. Rep. 1998, 31, 231-326.

(13) Nolte, P.; Stierle, A.; Kasper, N.; Jin-Phillipp, N. Y.; Jeutter, N.; Dosch, $\mathrm{H}$. Reversible Shape Changes of Pd Nanoparticles on $\mathrm{MgO}$ (100). Nano Lett. 2011, 11, 4697-4700.

(14) Penner, S.; Bera, P.; Pedersen, S.; Ngo, L. T.; Harris, J. J. W.; Campbell, C. T. Interactions of O2 with Pd Nanoparticles on $\alpha$ Al2O3(0001) at Low and High O2 Pressures. J. Phys. Chem. B 2006, $110,24577-24584$.

(15) Højrup Hansen, K.; Šljivančanin, Ž.; Lægsgaard, E.; Besenbacher, F.; Stensgaard, I. Adsorption of $\mathrm{O} 2$ and $\mathrm{NO}$ on $\mathrm{Pd}$ nanocrystals supported on $\mathrm{Al} 2 \mathrm{O} 3 / \mathrm{NiAl}(100)$ : overlayer and edge structures. Surf. Sci. 2002, 505, 25-38.

(16) Schalow, T.; Brandt, B.; Starr, D. E.; Laurin, M.; Shaikhutdinov, S. K.; Schauermann, S.; Libuda, J.; Freund, H. J. Particle size dependent adsorption and reaction kinetics on reduced and partially oxidized Pd nanoparticles. Phys. Chem. Chem. Phys. 2007, 9, 13471361.

(17) Lundgren, E.; Kresse, G.; Klein, C.; Borg, M.; Andersen, J. N.; De Santis, M.; Gauthier, Y.; Konvicka, C.; Schmid, M.; Varga, P. TwoDimensional Oxide on Pd(111). Phys. Rev. Lett. 2002, 88, 246103.

(18) Stará, I.; Matolín, V. The influence of particle size on CO adsorption on Pd/alumina model catalysts. Surf. Sci. 1994, 313, 99106.

(19) Borroni-Bird, C. E.; Al-Sarraf, N.; Andersoon, S.; King, D. A. Single crystal adsorption calorimetry. Chem. Phys. Lett. 1991, 183, $516-520$.

(20) Fischer-Wolfarth, J.-H.; Hartmann, J.; Farmer, J. A.; FloresCamacho, J. M.; Campbell, C. T.; Schauermann, S.; Freund, H.-J. An improved single crystal adsorption calorimeter for determining gas adsorption and reaction energies on complex model catalysts. Rev. Sci. Instrum. 2011, 82, 024102-024101.

(21) Nepijko, S. A.; Klimenkov, M.; Adelt, M.; Kuhlenbeck, H.; Schlögl, R.; Freund, H. J. Structural Investigation of Palladium Clusters on $\gamma-\mathrm{AlO} 3(111) / \mathrm{NiAl}(110)$ with Transmission Electron Microscopy. Langmuir 1999, 15, 5309-5313.

(22) Krüger, S.; Vent, S.; Nörtemann, F.; Staufer, M.; Rösch, N. The average bond length in Pd clusters Pdn, $\mathrm{n}=4-309$ : A densityfunctional case study on the scaling of cluster properties. J. Chem. Phys. 2001, 115, 2082-2087.

(23) Yudanov, I. V.; Metzner, M.; Genest, A.; Rösch, N. SizeDependence of Adsorption Properties of Metal Nanoparticles: A Density Functional Study on Palladium Nanoclusters. J. Phys. Chem. C 2008, 112, 20269-20275.

(24) Shustorovich, E.; Sellers, H. The UBI-QEP method: A practical theoretical approach to understanding chemistry on transition metal surfaces. Surf. Sci. Rep. 1998, 31, 1-119.

(25) Peter, M.; Flores Camacho, J. M.; Adamovski, S.; Ono, L. K.; Dostert, K.-H.; O’Brien, C. P.; Roldan Cuenya, B.; Schauermann, S.; Freund, H.-J. Trends in the Binding Strength of Surface Species on 
Nanoparticles: How Does the Adsorption Energy Scale with the Particle Size? Angew. Chem., Int. Ed. 2013, 52, 5175-5179.

(26) Note that the local adsorption environment (adsorption at the edge site) is preserved for all Pd nanoparticles and only the particle size changes.

(27) Daley, S. P.; Utz, A. L.; Trautman, T. R.; Ceyer, S. T. Ethylene Hydrogenation on $\mathrm{Ni}(111)$ by Bulk Hydrogen. J. Am. Chem. Soc. 1994, 116, 6001-6002.

(28) Teschner, D.; Borsodi, J.; Wootsch, A.; Révay, Z.; Hävecker, M.; Knop-Gericke, A.; Jackson, S. D.; Schlögl, R. The Roles of Subsurface Carbon and Hydrogen in Palladium-Catalyzed Alkyne Hydrogenation. Science 2008, 320, 86-89.

(29) Wilde, M.; Fukutani, K.; Ludwig, W.; Brandt, B.; Fischer, J.-H.; Schauermann, S.; Freund, H.-J. Influence of Carbon Deposition on the Hydrogen Distribution in Pd Nanoparticles and Their Reactivity in Olefin Hydrogenation. Angew. Chem., Int. Ed. 2008, 47, 9289-9293.

(30) Ludwig, W.; Savara, A.; Schauermann, S.; Freund, H.-J. Role of Low-Coordinated Surface Sites in Olefin Hydrogenation: A Molecular Beam Study on Pd Nanoparticles and Pd(111). ChemPhysChem 2010, 11, 2319-2322.

(31) Christmann, K. Interaction of hydrogen with solid surfaces. Surf. Sci. Rep. 1988, 9, 1-163.

(32) Hydrogen in metals; Alefeld, G., Volkl, J., Eds.; Springer-Verlag: Berlin, 1978.

(33) Greeley, J.; Mavrikakis, M. Surface and Subsurface Hydrogen: Adsorption Properties on Transition Metals and Near-Surface Alloys. J. Phys. Chem. B 2005, 109, 3460-3471.

(34) Wilde, M.; Fukutani, K.; Naschitzki, M.; Freund, H. J. Hydrogen absorption in oxide-supported palladium nanocrystals. Phys. Rev. B: Condens. Matter Mater. Phys. 2008, 77, 113412.

(35) Brandt, B.; Fischer, J.-H.; Ludwig, W.; Libuda, J.; Zaera, F.; Schauermann, S.; Freund, H.-J. Isomerization and Hydrogenation of cis-2-Butene on Pd Model Catalyst. J. Phys. Chem. C 2008, 112, $11408-11420$.

(36) Viñes, F.; Loschen, C.; Illas, F.; Neyman, K. M. Edge sites as a gate for subsurface carbon in palladium nanoparticles. J. Catal. 2009, 266, 59-63.

(37) Ludwig, W.; Savara, A.; Madix, R. J.; Schauermann, S.; Freund, H.-J. Subsurface Hydrogen Diffusion into Pd Nanoparticles: Role of Low-Coordinated Surface Sites and Facilitation by Carbon. J. Phys. Chem. C 2012, 116, 3539-3544.

(38) Neyman, K. M.; Schauermann, S. Hydrogen Diffusion into Palladium Nanoparticles: Pivotal Promotion by Carbon. Angew. Chem., Int. Ed. 2010, 49, 4743-4746. 